\title{
Review: cognitive behavioural interventions may be effective for chronic fatigue syndrome and chronic back pain
}

Raine R, Haines A, Sensky T.Systematic review of mental health interventions for patients with common somatic symptoms: can research evidence from secondary care be extrapolated to primary care? BMJ 2002 Nov;325:1082-93.

\section{QUESTION: Are mental health interventions for people with common somatic conditions effective? Can results from secondary care be extrapolated to primary care?}

\section{Design}

Systematic review with meta-analysis.

\section{Data sources}

Studies were identified using PubMed, the Cochrane Library, PsychLit and Embase (1966 - September 2001); reference lists; key texts, and citations from experts.

\section{Study selection}

Narrative systematic reviews, meta-analyses and randomised trials published in English were eligible if they assessed the effectiveness of primary or secondary care mental health interventions for chronic fatigue syndrome, irritable bowel syndrome or chronic back pain (where no physical cause was established). Eligible interventions included cognitive behavioural, cognitive, behaviour, brief interpersonal psychodynamic and antidepressant therapies. The authors identified 20 studies in primary care, 41 studies in secondary care and 2 meta-analyses.

\section{Data extraction}

Reviewers extracted data on participant characteristics, sample size, sample source, intervention, outcomes, study dropouts and reasons for withdrawal. Main outcome measures were reported health status and functional outcomes, treatment effect size and disease severity. The authors calculated treatment effects using fixed and random effects models.

\section{Main results}

Chronic fatigue syndrome: There is some evidence that cognitive behavioural therapy and behavioural therapy are effective in chronic fatigue syndrome. The authors found no evidence that brief psychodynamic interpersonal therapy or antidepressants improve symptoms in chronic fatigue syndrome.

Irritable bowel syndrome: There is some evidence that antidepressants and brief interpersonal therapy are effective in irritable bowel syndrome. There is limited evidence for the effectiveness of relaxation training and mixed evidence about the benefits of cognitive behaviour therapy.

Back pain: There is some evidence that cognitive behaviour therapy and behaviour therapy are effective for people with back pain. The authors found insufficient evidence on antidepressants or brief interpersonal therapy.

For all 3 syndromes, treatment effect sizes were largest in secondary care.

\section{Conclusions}

Mental health interventions may be effective for chronic fatigue syndrome, irritable bowel syndrome and chronic back pain. There is more evidence for the effectiveness of secondary care interventions than interventions in primary care.

\section{COMMENTARY}

Psychological disorders have a high financial burden with many indirect costs. Behavioural strategies and cognitive behavioural interventions may be effective for a range of mental disorders, including some of the most chronic, severe and costly mental health problems. ${ }^{1}$ Very few medical professionals are adequately trained to deliver such treatments, however. This article is both timely and important because it emphasises the need to disseminate cognitive behaviour therapies more widely.

Raine $e$ t al conducted a thorough review of the efficacy of psychological treatments for common somatic symptoms: chronic fatigue syndrome, irritable bowel syndrome and chronic back pain. The results were consistent with the findings by the American Psychological Association's Task Force on Promotion and Dissemination of Psychological Procedures: ${ }^{2}$ cognitive behaviour interventions and behaviour therapy are effective for treating chronic back pain and chronic fatigue syndrome. Raine et al found that treatment effects were stronger in secondary care compared with primary care settings. Furthermore, antidepressants were effective in both settings for treating irritable bowel syndrome.

The review has some limitations. First, as in all secondary analyses, the review is based only on published studies (that are more likely to report positive outcomes). There may also be problems with how interventions were defined and implemented. The majority of studies did not follow a treatment manual and did not measure adherence to the therapy protocol. The distinction between "behaviour therapy" and "cognitive-behaviour therapy" therefore remains elusive. This leaves important questions unanswered about how and why these treatments work (ie the mechanisms and mediators of change).

Stefan G Hofmann, $\mathrm{PhD}$ Department of Psychology Boston University Massachusetts, USA

1 Hofmann SG, Tompson MT. Treating chronic and severe mental disorders: A clinical handbook of empirically supported treatments. New York: Guilford Press.

2 Chambless DL, Sanderson, WC, Shoham V et al. An update on empirically validated treatments. Clin Psychologist 1996; 49: $5-18$

3 Kraemer HC, Wilson T, Fairburn CG, Agras WS. Mediators and moderators of treatment effects in randomized clinical trials. Arch Gen Psychiatry 2002: 59: 877-83.
Source of funding: UK Medical Research Council.

For correspondence: $R$ Raine, Department of Public Health and Policy, London School of Hygiene and Tropical Medicine, UK rosalind.raine@ lshtm.ac.uk 